tion mixture, the concentration of fluorescing particles could be studied as a function of time and the autocorrelation function formed. The kinetic constants derived again were in good agreement with relaxation spectroscopy results.

In variety there is spice. New information can be derived from experiments in which external fields are imposed on a fluctuating system at thermal equilibrium. Ware and Flygare used electrophoretic scattering ( $J$. Coll. Interf. Sci., 39, 670; 1972) in which charge-carrying scattering particles (in random Brownian motion) acquire and additional linear momentum by an applied d.c. electric field. In this situation the spectrum, or the autocorrelation function, can be clearly decomposed into contributions coming from the random, Brownian motion, and from the electrophoretic mobility of the particles. The resolution of the method is thus that, in priniciple, the motion of different particles, moving with different velocities, can be clearly distinguished. Hydrodynamic and sedimentation fields can, in principle, also be applied. In recent applications, Mustacich and Ware (Phys. Rev. Lett., 33, 617 ; 1974) reported on the study of protoplasmic streaming by laser light scattering and Josefowicz and Hallet (Appl. Optics, 14, 740; 1975) described a sophisticated electrophoretic light scattering assembly suitable for analysis at low sample concentrations.

Clegg, Elson and Maxfield (Biopolymers, 14, 883; 1975) introduced a new method in which a reaction is perturbed by repetitive application of a small pressure change. Reaction progress is followed by monitoring optical properties such as absorbance, fluorescence or light scattering. Phase-sensitive detection methods are used to enhance the low signal-to-noise ratio resulting from the slight perturbation. Yet the slight perturbation allows the analysis of closely spaced microstates, whereas the conventional perturbation methods, such as temperature-jump, often cause the system to transverse many intermediate conformations in the course of a single observation. This is of particular importance in reactions that involve the folding of biological macromolecules, in processes involving a multitude of partially ordered intermediate conformations.

In conclusion, a number of new tools for the study of reaction rates in chemical and biological reactions are being developed. In these new techniques advantage is taken of the spontaneous fluctuations around equilibrium states in the systems under study and, in other instances, only minor perturbations are superimposed on these natural fluctuations.

\section{Piezoelectric polyvinylidene fluoride}

\section{from Paul Calvert}

THERE has recently been considerable interest in the development of piezoelectric (producing a voltage when stretched) polymer films. The current champion is polyvinylidene fluoride (PVDF $\left.\left(\mathrm{CH}_{2}-\mathrm{CF}_{2}\right)_{\mathrm{n}}\right)$ which has a piezoelectric modulus $d_{31}$ of $1-2 \times 10^{-7}$ c.g.s.e.s.u. compared to about $18 \times 10^{-7}$ for barium titanate. The polymer, having the advantage of being available as a flexible film, is ready for use in microphones and possibly in loudspeakers. The origin of the piezoelectric effect in PVDF is still unknown and the matter is considerably confused by the complicated phase behaviour of this polymer. A pair of papers by Murayama and coworkers (J. Polymer Sci. Physics Edition, 13, 929 and 946; 1975) of the Kureha Chemical Company in Japan, one of the two commercial producers of this polymer, go a long way towards clarifying the question if not to producing answers.

In 1969 Fukada (Jap. J. appl. Phys., 8, 960) and Kawai (ibid., 975) showed that a piezoelectric effect could be produced in stretched and oriented films of several polymers when they were poled by applying an electric field of $500 \mathrm{kV} \mathrm{cm}^{-1}$ across the thickness of the film. When poled most polymers acquire a stable surface charge and are known as electrets. Except for PVDF there is no piezoelectric effect if the polymers are unoriented (Cohen and Edelman, J. appl. Phys., 42, 3072; 1971). This seems reasonable because the orientation process will tend to produce extended chain conformations of the polymer and in the vinyl polymers this would result in alignment of the permanent dipoles such as that of the $-\mathrm{CF}_{2}-$ group in $\mathrm{PVDF}$. If the electric field caused these to point preferentially in one direction the material could be piezoelectric.

What makes PVDF special is its phase behaviour. There are three crystal forms: I, also known as $\beta$, II or $\alpha$, and III, each of which can be produced by precipitation from a suitable solvent. Cooling from the melt gives form II which converts to form I on stretching. In form I the chains are extended so that the dipoles within one chain are parallel and the chains are packed into an orthorhombic lattice so that the dipoles on individual chains are parallel (Lando et al., J. Polymer Sci., A-1, 4, 941; 1966). Obviously this form should be piezoelectric if it is properly oriented. Doll and Lando $(J$. macromolec. Sci. Phys., 2, 205; 1968) showed that copolymerisation of tetra- fluorethylene $\left(\mathrm{CF}_{2}=\mathrm{CF}_{2}\right)$ with the vinylidene fluoride promoted formation of phase I and suggested that the 'head to head' groups $\left(-\mathrm{CH}_{2} \mathrm{CF}_{2} \mathrm{CF}_{2} \mathrm{CH}_{2}\right)$ normally present at $5-10 \%$ in PVDF promote form I. Tadokoro et al. (Macromolecules, $\mathbf{8}, 158 ; 1975$ ) go into this in more detail with infrared and Raman spectra and discuss the structures of form II, where the dipoles cancel, and form III which is similar to form I.

Hayakawa and Wada (Adv. Polymer Sci., 11, 1; 1973) have discussed the possible origins of piezoelectric effects in polymers and distinguish between intrinsic effects due to oriented dipoles and extrinsic effects due to trapped charges. On the face of it the former looks more likely for PVDF where the piezoelectric modulus increases with the content of form I whether produced by stretching or copolymerisation. Since the dipoles in form $\mathrm{I}$ are aligned it is simply necessary to assume that the crystals realign in the electric field or that more form I arises during the poling.

One observation that clashes with this is that of a negative piezoelectric modulus $d_{33}$ in the film thickness direction by Burkard and Pfister ( $J$. appl. Phys., 45, 3360; 1974). Any simple dipole model would predict a positive value; that is the film should get thicker when a field is applied parallel to the original poling field.

Murayama et al. report measurements on the decay of surface charge after poling. PVDF electrets have a charge of the same polarity as the field (homocharge) which is probably due to charges injected from the electrodes. This decays over a few hours to leave a charge of opposite sign (anomalous heterocharge) which is stable, even above the poling temperature. This anomalous heterocharge is correlated with, but not the origin of, the piezoelectric effect. They also observe that if a polyester film is placed between the positive electrode and the PVDF during poling the piezoelectric modulus and heterocharge are much reduced; between the negative electrode and the film it has little effect. No evidence is found for the production of more form I on poling and they argue that reorientation of existing crystals is very unlikely. Thus they conclude from their observations that the piezoelectric effect cannot be due to permanent dipoles but must be caused by the local migration and trapping of charges in the form I crystals. This process is somehow associated with charge injection from the positive electrode. The matter cannot really be considered settled until more is known about the nature of the induced dipoles and why form I in PVDF is particularly suitable. By then there will probably be a new champion polymer. 